\title{
Common Fixed Points for Two Contractive Mappings of Integral Type in Metric Spaces
}

\author{
Xing Jin, Yongjie Piao* \\ Department of Mathematics, College of Science, Yanbian University, Yanji, China \\ Email: sxpyj@ybu.edu.cn \\ Received 12 May 2015; accepted 2 June 2015; published 5 June 2015 \\ Copyright (C) 2015 by authors and Scientific Research Publishing Inc. \\ This work is licensed under the Creative Commons Attribution International License (CC BY). \\ http://creativecommons.org/licenses/by/4.0/

c. (i) Open Access

\section{Abstract}

In this paper, we obtain unique common fixed point theorems for two mappings satisfying the variable coefficient linear contraction of integral type and the implicit contraction of integral type respectively in metric spaces.

\section{Keywords}

\section{Contractive Mapping of Integral Type, Common Fixed Point, Metric Space}

\section{Introduction and Preliminaries}

Throughout this paper, we assume that $R^{+}=[0, \infty)$ and $\Phi=\left\{\phi \mid \phi: R^{+} \rightarrow R^{+}\right.$satisfying that $\phi$ is Lebesgue integral, summable on each compact subset of $R^{+}$and $\int_{0}^{\varepsilon} \phi(t) \mathrm{d} t>0$ for each $\left.\varepsilon>0\right\}$.

The famous Banach's contraction principle is as follows:

Theorem 1.1 ([1]). Let $f$ be a self mapping on a complete metric space $(X, d)$ satisfying

$$
d(f x, f y) \leq c d(x, y) \text { for all } x, y \in X,
$$

where $c \in[0,1)$ is a constant. Then $f$ has a unique fixed point $\tilde{x} \in X$ such that $\lim _{n \rightarrow \infty} f^{n}(x)=\tilde{x}$ for each $x \in X$.

It is known that the Banach contraction principle has a lot of generalizations and various applications in many directions; see, for examples, [2]-[15] and the references cited therein. In 1962, Rakotch [11] extended the Banach contraction principle with replacing the contraction constant $c$ in (1.1) by a contraction function $\gamma$ and obtained the next theorem.

Theorem 1.2 ([11]). Let $f$ be a self-mapping on a complete metric space $(X, d)$ satisfying

${ }^{*}$ Corresponding author. 


$$
d(f x, f y) \leq \gamma(d(x, y)) d(x, y) \text { for all } x, y \in X,
$$

where $\gamma: R^{+} \rightarrow[0,1)$ is a monotonically decreasing function. Then $f$ has a unique fixed point $\tilde{x} \in X$ such that $\lim _{n \rightarrow \infty} f^{n}(x)=\tilde{x}$ for each $x \in X$.

In 2002, Branciari [12] gave an integral version of Theorem 1.1 as follows.

Theorem 1.3 ([12]). Let $f$ be a self-mapping on a complete metric space $(X, d)$ satisfying

$$
\int_{0}^{d(f x, f y)} \phi(t) \mathrm{d} t \leq c \int_{0}^{d(x, y)} \phi(t) \mathrm{d} t \text { for all } x, y \in X,
$$

where $c \in[0,1)$ is a constant and $\phi \in \Phi$. Then $f$ has a unique fixed point $\tilde{x} \in X$ such that $\lim _{n \rightarrow \infty} f^{n}(x)=\tilde{x}$ for each $x \in X$.

In 2011, Liu and Li [13] modified the method of Rakotch to generalize the Branciari's fixed point theorem with replacing the contraction constant $c$ in (1.3) by contraction functions $\alpha$ and $\beta$ and established the following fixed point theorem:

Theorem 1.4 ([13]). Let $f$ be a self-mapping on a complete metric space $(X, d)$ satisfying

$$
\int_{0}^{d(f x, f y)} \phi(t) \mathrm{d} t \leq \alpha(d(x, y)) \int_{0}^{d(x, f x)} \phi(t) \mathrm{d} t+\beta(d(x, y)) \int_{0}^{d(y, f y)} \phi(t) \mathrm{d} t \text { for all } x, y \in X,
$$

where $\phi \in \Phi$ and $\alpha, \beta: R^{+} \rightarrow[0,1)$ are two functions with $\alpha(t)+\beta(t)<1$ for all $t \in R^{+} ; \limsup _{s \rightarrow 0^{+}} \beta(s)<1 ; \limsup _{s \rightarrow t^{+}} \frac{1-\alpha(s)}{\beta(s)}<1$ for all $t>0$.

Then $f$ has a unique fixed point $\tilde{x} \in X$ such that $\lim _{n \rightarrow \infty} f^{n}(x)=\tilde{x}$ for each $x \in X$.

Here, we will use the methods in [3] [9] [13] to discuss the unique existence problems of common fixed points for two self-mappings satisfying two different contractive conditions of integral type in a complete metric space.

\section{Common Fixed Point Theorems}

Lemma 2.1 ([13]). Let $\phi \in \Phi$ and $\left\{r_{n}\right\}_{n \in N}$ be a nonnegative sequence with $\lim _{n \rightarrow \infty} r_{n}=r$. Then

$$
\lim _{n \rightarrow \infty} \int_{0}^{r_{n}} \phi(t) \mathrm{d} t=\int_{0}^{r} \phi(t) \mathrm{d} t .
$$

Lemma 2.2([13]). Let $\phi \in \Phi$ and $\left\{r_{n}\right\}_{n \in N}$ be a nonnegative sequence. Then

$$
\lim _{n \rightarrow \infty} \int_{0}^{r_{n}} \phi(t) \mathrm{d} t=0 \Leftrightarrow \lim _{n \rightarrow \infty} r_{n}=0 .
$$

Now, we will give the first main result in this paper.

Theorem 2.1. Let $(X, d)$ be a complete metric space, $f, g: X \rightarrow X$ two mappings. If for each $x, y \in X$,

$$
\begin{aligned}
\int_{0}^{d(f x, g y)} \phi(t) \mathrm{d} t \leq & \alpha(d(x, y)) \int_{0}^{d(x, y)} \phi(t) \mathrm{d} t+\beta(d(x, y)) \int_{0}^{d(x, f x)} \phi(t) \mathrm{d} t \\
& +\gamma(d(x, y)) \int_{0}^{d(y, g y)} \phi(t) \mathrm{d} t,
\end{aligned}
$$

where $\phi \in \Phi$ and $\alpha, \beta, \gamma: R^{+} \rightarrow[0,1)$ are three functions satisfying the following conditions

$$
\begin{gathered}
\alpha(t)+\beta(t)+\gamma(t)<1 \text { for all } t \in[0, \infty), \max \left\{\limsup _{s \rightarrow 0^{+}} \beta(s), \limsup _{s \rightarrow 0^{+}} \gamma(s)\right\}<1 \\
\max \left\{\limsup _{s \rightarrow t^{+}} \alpha(t), \limsup _{s \rightarrow t^{+}} \frac{\alpha(t)+\gamma(t)}{1-\beta(t)}, \limsup _{s \rightarrow t^{+}} \frac{\alpha(t)+\beta(t)}{1-\gamma(t)}\right\}<1 \text { for all } t \in[0, \infty) .
\end{gathered}
$$

Then $f$ and $g$ have a unique common fixed point $u$, and the sequence $\left\{x_{n}\right\}_{n \in N}$ defined by $x_{2 n+1}=f x_{2 n}$, $x_{2 n+2}=g x_{2 n+1}$ for any $x_{0} \in X$ converges to $u$.

Proof.

Let $x_{0}=x \in X$. We construct a sequence $\left\{x_{n}\right\}_{n \in N}$ satisfying the following conditions $x_{2 n+1}=f x_{2 n}$, $x_{2 n+2}=g x_{2 n+1}$ for all $n \in N \bigcup\{0\}$. Let $d_{n}=d\left(x_{n}, x_{n+1}\right)$ for all $n \in N \bigcup\{0\}$.

For $n \in N \cup\{0\}$, by (2.1), 


$$
\begin{aligned}
& \int_{0}^{d_{2 n}} \phi(t) \mathrm{d} t \\
& =\int_{0}^{d\left(f x_{2 n}, g x_{2 n-1}\right)} \phi(t) \mathrm{d} t \leq \alpha\left(d\left(x_{2 n}, x_{2 n-1}\right)\right) \int_{0}^{d\left(x_{2 n}, x_{2 n-1}\right)} \phi(t) \mathrm{d} t \\
& \quad+\beta\left(d\left(x_{2 n}, x_{2 n-1}\right)\right) \int_{0}^{d\left(x_{2 n}, f_{2 n}\right)} \phi(t) \mathrm{d} t+\gamma\left(d\left(x_{2 n}, x_{2 n-1}\right)\right) \int_{0}^{d\left(x_{2 n-1}, g x_{2 n-1}\right)} \phi(t) \mathrm{d} t \\
& =\left[\alpha\left(d\left(x_{2 n}, x_{2 n-1}\right)\right)+\gamma\left(d\left(x_{2 n}, x_{2 n-1}\right)\right)\right] \int_{0}^{d_{2 n-1}} \phi(t) \mathrm{d} t+\beta\left(d\left(x_{2 n}, x_{2 n-1}\right)\right) \int_{0}^{d_{2 n}} \phi(t) \mathrm{d} t,
\end{aligned}
$$

hence by (2.3),

$$
\int_{0}^{d_{2 n}} \phi(t) \mathrm{d} t \leq \frac{\alpha\left(d\left(x_{2 n}, x_{2 n-1}\right)\right)+\gamma\left(d\left(x_{2 n}, x_{2 n-1}\right)\right)}{1-\beta\left(d\left(x_{2 n}, x_{2 n-1}\right)\right)} \int_{0}^{d_{2 n-1}} \phi(t) \mathrm{d} t \leq \int_{0}^{d_{2 n-1}} \phi(t) \mathrm{d} t .
$$

Similarly, by (2.1),

$$
\begin{aligned}
\int_{0}^{d_{2 n+1}} \phi(t) \mathrm{d} t= & \int_{0}^{d\left(f_{2 n}, g x_{2 n+11}\right)} \phi(t) \mathrm{d} t \\
\leq & {\left[\alpha\left(d\left(x_{2 n}, x_{2 n+1}\right)\right)+\beta\left(d\left(x_{2 n}, x_{2 n+1}\right)\right)\right] \int_{0}^{d_{2 n}} \phi(t) \mathrm{d} t } \\
& +\gamma\left(d\left(x_{2 n}, x_{2 n+1}\right)\right) \int_{0}^{d_{2 n+1}} \phi(t) \mathrm{d} t,
\end{aligned}
$$

hence by (2.3),

$$
\int_{0}^{d_{2 n+1}} \phi(t) \mathrm{d} t \leq \frac{\alpha\left(d\left(x_{2 n}, x_{2 n+1}\right)\right)+\beta\left(d\left(x_{2 n}, x_{2 n+1}\right)\right)}{1-\gamma\left(d\left(x_{2 n}, x_{2 n+1}\right)\right)} \int_{0}^{d_{2 n}} \phi(t) \mathrm{d} t \leq \int_{0}^{d_{2 n}} \phi(t) \mathrm{d} t .
$$

Combining (2.4) and (2.5), we have

$$
\int_{0}^{d_{n+1}} \phi(t) \mathrm{d} t \leq \int_{0}^{d_{n}} \phi(t) \mathrm{d} t \text { for all } n \in N \bigcup\{0\} .
$$

Now, we prove that

$$
d_{n+1} \leq d_{n} \text { for all } n \in N \bigcup\{0\} .
$$

Otherwise, there exists $n_{0} \in N$ such that

$$
d_{n_{0}}>d_{n_{0}-1} .
$$

Obviously, $d_{n_{0}}>0$. If $n_{0}=2 k$, then by (2.3), (2.4), (2.6) and (2.8),

$$
\begin{aligned}
0 & <\int_{0}^{d_{n_{0}}} \phi(t) \mathrm{d} t \leq \int_{0}^{d_{n_{0}-1}} \phi(t) \mathrm{d} t \leq \int_{0}^{d_{n_{0}}} \phi(t) \mathrm{d} t=\int_{0}^{d_{2 k}} \phi(t) \mathrm{d} t \\
& \leq \frac{\alpha\left(d\left(x_{2 k}, x_{2 k-1}\right)\right)+\gamma\left(d\left(x_{2 k}, x_{2 k-1}\right)\right)}{1-\beta\left(d\left(x_{2 k}, x_{2 k-1}\right)\right)} \int_{0}^{d_{2 k-1}} \phi(t) \mathrm{d} t \\
& <\int_{0}^{d_{2 k-1}} \phi(t) \mathrm{d} t=\int_{0}^{d_{n_{0}}-1} \phi(t) \mathrm{d} t
\end{aligned}
$$

which is a contradiction. Similarly, if $n_{0}=2 k+1$, then by (2.3), (2.5), (2.6) and (2.8),

$$
\begin{aligned}
0 & <\int_{0}^{d_{n_{0}}} \phi(t) \mathrm{d} t \leq \int_{0}^{d_{n_{0}-1}} \phi(t) \mathrm{d} t \leq \int_{0}^{d_{n_{0}}} \phi(t) \mathrm{d} t=\int_{0}^{d_{2 k+1}} \phi(t) \mathrm{d} t \\
& \leq \frac{\alpha\left(d\left(x_{2 k}, x_{2 k+1}\right)\right)+\beta\left(d\left(x_{2 k}, x_{2 k+1}\right)\right)}{1-\gamma\left(d\left(x_{2 k}, x_{2 k+1}\right)\right)} \int_{0}^{d_{2 k}} \phi(t) \mathrm{d} t<\int_{0}^{d_{2 k}} \phi(t) \mathrm{d} t=\int_{0}^{d_{n_{0}-1}} \phi(t) \mathrm{d} t
\end{aligned}
$$

which is also a contradiction. Hence (2.7) holds. Therefore there exists $u \geq 0$ such that $\lim _{n \rightarrow \infty} d_{n}=u$. If $u>0$, then by Lemma 2.1, (2.3) and (2.4), 


$$
\begin{aligned}
0 & <\int_{0}^{u} \phi(t) \mathrm{d} t=\lim _{n \rightarrow \infty} \int_{0}^{d_{2 n}} \phi(t) \mathrm{d} t \\
& \leq \limsup \left[\frac{\alpha\left(d\left(x_{2 k}, x_{2 k-1}\right)\right)+\gamma\left(d\left(x_{2 k}, x_{2 k-1}\right)\right)}{1-\beta\left(d\left(x_{2 k}, x_{2 k-1}\right)\right)} \int_{0}^{d_{2 n-1}} \phi(t) \mathrm{d} t\right] \\
& \leq \limsup \left[\frac{\alpha\left(d\left(x_{2 k}, x_{2 k-1}\right)\right)+\gamma\left(d\left(x_{2 k}, x_{2 k-1}\right)\right)}{1-\beta\left(d\left(x_{2 k}, x_{2 k-1}\right)\right)}\right] \limsup \operatorname{sum}_{n \rightarrow \infty} \int_{0}^{d_{2 n-1}} \phi(t) \mathrm{d} t \\
& <\int_{0}^{u} \phi(t) \mathrm{d} t
\end{aligned}
$$

which is a contradiction. Therefore $u=0$, that is, $\lim _{n \rightarrow \infty} d_{n}=0$.

We claim that $\left\{x_{n}\right\}_{n \in N}$ is a Cauchy sequence. Otherwise, there $\varepsilon>0$ such that for $k \in N$, there exist $m(k), n(k) \in N$ with $m(k)>n(k)$ such that the parity of $m(k)$ and $n(k)$ is different and

$$
d\left(x_{m(k)}, x_{n(k)}\right)>\varepsilon \text {. }
$$

For $k$, let $m(k)$ denotes the least integer exceeding $n(k)$ and satisfying the above, then

$$
d\left(x_{m(k)}, x_{n(k)}\right)>\varepsilon, d\left(x_{m(k)-2}, x_{n(k)}\right) \leq \varepsilon \text {, for all } k \in N,
$$

hence

$$
\varepsilon<d\left(x_{m(k)}, x_{n(k)}\right) \leq d\left(x_{n(k)}, x_{m(k)-2}\right)+d_{m(k)-2}+d_{m(k)-1} \leq \varepsilon+d_{m(k)-2}+d_{m(k)-1} .
$$

Let $k \rightarrow \infty$, then we obtain $\varepsilon=\lim _{k \rightarrow \infty} d\left(x_{m(k)}, x_{n(k)}\right)=\lim _{k \rightarrow \infty} d\left(x_{m(k)-2}, x_{n(k)}\right)$. But

$$
\begin{aligned}
& \left|d\left(x_{m(k)+1}, x_{n(k)}\right)-d\left(x_{m(k)}, x_{n(k)}\right)\right| \leq d_{m(k)}, \\
& \left|d\left(x_{m(k)+1}, x_{n(k)+1}\right)-d\left(x_{m(k)+1}, x_{n(k)}\right)\right| \leq d_{n(k)}, \\
& \left|d\left(x_{m((k)}, x_{n(k)}\right)-d\left(x_{m(k)}, x_{n(k)+1}\right)\right| \leq d_{n(k)},
\end{aligned}
$$

hence we obtain

$$
\begin{aligned}
\varepsilon & =\lim _{k \rightarrow \infty} d\left(x_{m(k)}, x_{n(k)}\right)=\lim _{k \rightarrow \infty} d\left(x_{m(k)}, x_{n(k)+1}\right) \\
& =\lim _{k \rightarrow \infty} d\left(x_{m(k)+1}, x_{n(k)}\right)=\lim _{k \rightarrow \infty} d\left(x_{m(k)+1}, x_{n(k)+1}\right) .
\end{aligned}
$$

If $m(k)$ is even and $n(k)$ is odd, then by Lemma 2.1, (2.11) and (2.1),

$$
\begin{aligned}
0< & \int_{0}^{\varepsilon} \phi(t) \mathrm{d} t=\limsup _{k \rightarrow \infty} \int_{0}^{d\left(x_{m(k)+1}, x_{n(k)+1}\right)} \phi(t) \mathrm{d} t=\lim \sup _{k \rightarrow \infty} \int_{0}^{d\left(f x_{m(k)}, g x_{n(k)}\right)} \phi(t) \mathrm{d} t \\
\leq & \limsup _{k \rightarrow \infty}\left[\alpha\left(d\left(x_{m(k)}, x_{n(k)}\right)\right) \int_{0}^{d\left(x_{m(k)}, x_{n(k)}\right)} \phi(t) \mathrm{d} t\right. \\
& \left.+\beta\left(d\left(x_{m(k)}, x_{n(k)}\right)\right) \int_{0}^{d\left(x_{m(k)}, f x_{m(k)}\right)} \phi(t) \mathrm{d} t+\gamma\left(d\left(x_{m(k)}, x_{n(k)}\right)\right) \int_{0}^{d\left(x_{n(k)}, g x_{n(k)}\right)} \phi(t) \mathrm{d} t\right] \\
= & \limsup \sup _{k \rightarrow \infty}\left[\alpha\left(d\left(x_{m(k)}, x_{n(k)}\right)\right) \int_{0}^{d\left(x_{m(k)}, x_{n(k)}\right)} \phi(t) \mathrm{d} t\right. \\
& \left.+\beta\left(d\left(x_{m(k)}, x_{n(k)}\right)\right) \int_{0}^{d}{ }_{m(k)} \phi(t) \mathrm{d} t+\gamma\left(d\left(x_{m(k)}, x_{n(k)}\right)\right) \int_{0}^{d_{n(k)}} \phi(t) \mathrm{d} t\right] \\
\leq & \lim \sup _{s \rightarrow \varepsilon} \alpha(s) \int_{0}^{\varepsilon} \phi(t) \mathrm{d} t\left(\because d_{n(k)} \rightarrow 0, d_{m(k)} \rightarrow 0\right)<\int_{0}^{\varepsilon} \phi(t) \mathrm{d} t
\end{aligned}
$$

which is a contradiction. Similarly, we obtain the same contradiction for the case that $m(k)$ is odd and $n(k)$ is even. Hence $\left\{x_{n}\right\}$ is a Cauchy sequence, therefore $\lim _{n \rightarrow \infty} x_{n}=x^{*}$ for some $x^{*} \in X$ by the completeness of $X$.

If $f x^{*} \neq x^{*}$, then $d\left(f x^{*}, x^{*}\right)>0$, hence by (2.1) and Lemma 2.1, 


$$
\begin{aligned}
0< & \int_{0}^{d\left(f x^{*}, x^{*}\right)} \phi(t) \mathrm{d} t=\limsup _{k \rightarrow \infty} \int_{0}^{d\left(f x^{*}, g x_{2 n+1}\right)} \phi(t) \mathrm{d} t \\
\leq & \limsup _{k \rightarrow \infty}\left[\alpha\left(d\left(x^{*}, x_{2 n+1}\right)\right) \int_{0}^{d\left(x^{*}, x_{2 n+1}\right)} \phi(t) \mathrm{d} t\right. \\
& \left.+\beta\left(d\left(x^{*}, x_{2 n+1}\right)\right) \int_{0}^{d\left(x^{*}, x^{*}\right)} \phi(t) \mathrm{d} t+\gamma\left(d\left(x^{*}, x_{2 n+1}\right)\right) \int_{0}^{d\left(x_{2 n+1}, g x_{2 n+1}\right)} \phi(t) \mathrm{d} t\right] \\
& \leq \limsup _{s \rightarrow 0^{+}} \beta(s) \int_{0}^{d\left(f x^{*}, x^{*}\right)} \phi(t) \mathrm{d} t\left(\because d\left(x^{*}, x_{2 n+1}\right) \rightarrow 0, d_{2 n+1} \rightarrow 0\right) \\
& <\int_{0}^{d\left(f x^{*}, x^{*}\right)} \phi(t) \mathrm{d} t
\end{aligned}
$$

which is a contradiction, hence $f x^{*}=x^{*}$. Similarly, we obtain $g x^{*}=x^{*}$. Therefore $x^{*}$ is a common fixed point of $f$ and $g$.

If $y^{*}$ is another common fixed point of $f$ and $g$, then $d\left(x^{*}, y^{*}\right)>0$, hence by (2.1),

$$
\begin{aligned}
0< & \int_{0}^{d\left(x^{*}, y^{*}\right)} \phi(t) \mathrm{d} t=\int_{0}^{d\left(f^{*}, g y^{*}\right)} \phi(t) \mathrm{d} t \\
\leq & \alpha\left(d\left(x^{*}, y^{*}\right)\right) \int_{0}^{d\left(x^{*}, y^{*}\right)} \phi(t) \mathrm{d} t+\beta\left(d\left(x^{*}, y^{*}\right)\right) \int_{0}^{d\left(x^{*}, f x^{*}\right)} \phi(t) \mathrm{d} t \\
& +\gamma\left(d\left(x^{*}, y^{*}\right)\right) \int_{0}^{d\left(y^{*}, g y^{*}\right)} \phi(t) \mathrm{d} t \\
= & \alpha\left(d\left(x^{*}, y^{*}\right)\right) \int_{0}^{d\left(x^{*}, y^{*}\right)} \phi(t) \mathrm{d} t<\int_{0}^{d\left(x^{*}, y^{*}\right)} \phi(t) \mathrm{d} t
\end{aligned}
$$

which is a contradiction, hence $x^{*}=y^{*}$, i.e., $x^{*}$ is the unique common fixed point of $f$ and $g$.

From Theorem 2.1, we obtain the next more general common fixed point theorem.

Theorem 2.2. Let $(X, d)$ be a complete metric space, $m, n \in N$ and $f, g: X \rightarrow X$ two mappings. If for each $x, y \in X$,

$$
\begin{aligned}
\int_{0}^{d\left(f^{m} x, g^{n} y\right)} \phi(t) \mathrm{d} t \leq & \alpha(d(x, y)) \int_{0}^{d(x, y)} \phi(t) \mathrm{d} t+\beta(d(x, y)) \int_{0}^{d\left(x, f^{m} x\right)} \phi(t) \mathrm{d} t \\
& +\gamma(d(x, y)) \int_{0}^{d\left(y, g^{n} y\right)} \phi(t) \mathrm{d} t
\end{aligned}
$$

where $\phi \in \Phi, \alpha, \beta, \gamma: R^{+} \rightarrow[0,1)$ are three functions satisfying (2.2) and (2.3). Then $f$ and $g$ have a unique common fixed point $u$, and the sequence $\left\{x_{k}\right\}_{k \in N}$ defined by $x_{2 n+1}=f^{m} x_{2 n}, x_{2 n+2}=g^{n} x_{2 n+1}$ for any $x_{0} \in X$ converges to $u$.

Proof.

Let $F=f^{m}$ and $G=g^{n}$, then $F$ and $G$ satisfy all of the conditions of Theorem 2.1, hence there exists an unique element $u \in X$ such that $f^{m} u=F u=u=G u=g^{n} u$. If $f u \neq u$, then $d(f u, u)>0$, hence by (2.12),

$$
0<\int_{0}^{d(f u, u)} \phi(t) \mathrm{d} t=\int_{0}^{d\left(f^{m} f u, g^{n} u\right)} \phi(t) \mathrm{d} t \leq \alpha(d(f u, u)) \int_{0}^{d(f u, u)} \phi(t) \mathrm{d} t<\int_{0}^{d(f u, u)} \phi(t) \mathrm{d} t
$$

which is a contradiction, hence $f u=u$. Similarly, $g u=u$. So $u$ is a common fixed point of $f$ and $g$. The uniqueness is obvious.

From now on, we will discuss the second common fixed point problem for two mappings with implicit contraction of integral type.

Let $\psi \in \Psi$ if and only if $\psi:\left(R^{+}\right)^{5} \rightarrow R^{+}$is a continuous and non-decreasing function about the 4th and 5th variables and satisfying the following conditions:

(i) There exists $h_{1} \in(0,1)$ such that $u \leq \psi(v, u, v, 0, u+v)$ implies $u \leq h_{1} v$;

(ii) There exists $h_{2} \in(0,1)$ such that $u \leq \psi(v, v, u, u+v, 0)$ implies $u \leq h_{2} v$;

(iii) $\psi(t, 0,0, t, t)<t, \psi(0, t, 0,0, t)<t, \psi(0,0, t, t, 0)<t$ for all $t>0$.

Example 2.1. Define $\psi:\left(R^{+}\right)^{5} \rightarrow R^{+}$as follows 


$$
\psi\left(x_{1}, x_{2}, x_{3}, x_{4}, x_{5}\right)=\sum_{i=1}^{5} a_{i} x_{i} \text { for all } x_{1}, x_{2}, x_{3}, x_{4}, x_{5} \in R^{+},
$$

where $a_{i} \geq 0$ for all $i=1,2,3,4,5$ and $a_{1}+a_{2}+a_{3}+2 a_{4}+2 a_{5}<1$. Then $\psi \in \Psi$.

The function $\phi \in \Phi$ is called to be sub-additive if and only if for all $a, b \geq 0$,

$$
\int_{0}^{a+b} \phi(t) \mathrm{d} t \leq \int_{0}^{a} \phi(t) \mathrm{d} t+\int_{0}^{b} \phi(t) \mathrm{d} t
$$

Example 2.2. Let $\phi(t)=\frac{1}{1+t}$ for each $t \geq 0$. Then obviously $\phi \in \Phi$ and for all $a, b \geq 0$,

$$
\int_{0}^{a+b} \phi(t) \mathrm{d} t=\ln ^{(1+a+b)} \leq \ln ^{(1+a)(1+b)}=\int_{0}^{a} \phi(t) \mathrm{d} t+\int_{0}^{b} \phi(t) \mathrm{d} t .
$$

Hence $\phi$ is a sub-additive function.

Theorem 2.3. Let $(X, d)$ be a complete metric space, $f, g: X \rightarrow X$ two mappings. If for each $x, y \in X$,

$$
\int_{0}^{d(f x, g y)} \phi(t) \mathrm{d} t \leq \psi\left(\int_{0}^{d(x, y)} \phi(t) \mathrm{d} t, \int_{0}^{d(x, f x)} \phi(t) \mathrm{d} t, \int_{0}^{d(y, g y)} \phi(t) \mathrm{d} t, \int_{0}^{d(x, g y)} \phi(t) \mathrm{d} t, \int_{0}^{d(f x, g)} \phi(t) \mathrm{d} t\right),
$$

where $\phi \in \Phi$ is sub-additive and $\psi \in \Psi$. Then $f$ and $g$ have a unique common fixed point.

\section{Proof.}

We take any element $x_{0} \in X$ and consider the sequence $\left\{x_{k}\right\}$ constructed by $x_{2 k+1}=f x_{2 k}$ and $x_{2 k+2}=g x_{2 k+1}$ for all $k \in N \cup\{0\}$. Let $d_{n}=d\left(x_{n}, x_{n+1}\right)$ for all $n \in N \cup\{0\}$.

Since

$$
\begin{aligned}
& \int_{0}^{d_{2 n}} \phi(t) \mathrm{d} t=\int_{0}^{d\left(f x_{2 n}, g x_{2 n-1}\right)} \phi(t) \mathrm{d} t \\
& \leq \psi\left(\int_{0}^{d\left(x_{2 n}, x_{2 n-1}\right)} \phi(t) \mathrm{d} t, \int_{0}^{d\left(x_{2 n}, f x_{2 n}\right)} \phi(t) \mathrm{d} t, \int_{0}^{d\left(x_{2 n-1}, g x_{2 n-1}\right)} \phi(t) \mathrm{d} t, \int_{0}^{d\left(x_{2 n}, g x_{2 n-1}\right)} \phi(t) \mathrm{d} t, \int_{0}^{d\left(f x_{2 n}, x_{2 n-1}\right)} \phi(t) \mathrm{d} t\right) \\
& \leq \psi\left(\int_{0}^{d_{2 n-1}} \phi(t) \mathrm{d} t, \int_{0}^{d_{2 n}} \phi(t) \mathrm{d} t, \int_{0}^{d_{2 n+1}} \phi(t) \mathrm{d} t, 0, \int_{0}^{d_{2 n-1}+d_{2 n}} \phi(t) \mathrm{d} t\right) \\
& \leq \psi\left(\int_{0}^{d_{2 n-1}} \phi(t) \mathrm{d} t, \int_{0}^{d_{2 n}} \phi(t) \mathrm{d} t, \int_{0}^{d_{2 n-1}} \phi(t) \mathrm{d} t, 0, \int_{0}^{d_{2 n-1}} \phi(t) \mathrm{d} t+\int_{0}^{d_{2 n}} \phi(t) \mathrm{d} t\right) .
\end{aligned}
$$

So by (i),

$$
\int_{0}^{d_{2 n}} \phi(t) \mathrm{d} t \leq h_{1} \int_{0}^{d_{2 n-1}} \phi(t) \mathrm{d} t \text { for all } n \in N \bigcup\{0\}
$$

Similarly,

$$
\begin{aligned}
& \int_{0}^{d_{2 n+1}} \phi(t) \mathrm{d} t=\int_{0}^{d\left(f x_{2 n}, g x_{2 n+1}\right)} \phi(t) \mathrm{d} t \\
& \leq \psi\left(\int_{0}^{d\left(x_{2 n}, x_{2 n+1}\right)} \phi(t) \mathrm{d} t, \int_{0}^{d\left(x_{2 n}, f x_{2 n}\right)} \phi(t) \mathrm{d} t, \int_{0}^{d\left(x_{2 n+1}, g x_{2 n+1}\right)} \phi(t) \mathrm{d} t, \int_{0}^{d\left(x_{2 n}, g x_{2 n+1}\right)} \phi(t) \mathrm{d} t, \int_{0}^{d\left(f x_{2 n}, x_{2 n+1}\right)} \phi(t) \mathrm{d} t\right) \\
& \leq \psi\left(\int_{0}^{d_{2 n}} \phi(t) \mathrm{d} t \int_{0}^{d_{2 n}} \phi(t) \mathrm{d} t, \int_{0}^{d_{2 n+1}} \phi(t) \mathrm{d} t, \int_{0}^{d_{2 n}+d_{2 n+1}} \phi(t) \mathrm{d} t, 0\right) \\
& \leq \psi\left(\int_{0}^{d_{2 n}} \phi(t) \mathrm{d} t, \int_{0}^{d_{2 n}} \phi(t) \mathrm{d} t, \int_{0}^{d_{2 n+1}} \phi(t) \mathrm{d} t, \int_{0}^{d_{2 n}} \phi(t) \mathrm{d} t+\int_{0}^{d_{2 n+1}} \phi(t) \mathrm{d} t, 0\right) .
\end{aligned}
$$

So by (ii),

$$
\int_{0}^{d_{2 n+1}} \phi(t) \mathrm{d} t \leq h_{2} \int_{0}^{d_{2 n}} \phi(t) \mathrm{d} t \quad \text { for all } n \in N \bigcup\{0\}
$$

Combining (2.14) and (2.15), we have

$$
\int_{0}^{d_{n+1}} \phi(t) \mathrm{d} t \leq \max \left\{h_{1}, h_{2}\right\} \int_{0}^{d_{n}} \phi(t) \mathrm{d} t<\int_{0}^{d_{n}} \phi(t) \mathrm{d} t \text { for all } n \in N \bigcup\{0\} .
$$

Obviously, $d_{n+1} \neq d_{n}$ for all $n \in N \cup\{0\}$. If there exits $n_{0}$ such that $d_{n_{0}}>d_{n_{0}-1}$, then $d_{n_{0}}>0$. If $n_{0}=2 k$, then by (2.14) and (2.16) 


$$
0<\int_{0}^{d_{n_{0}}} \phi(t) \mathrm{d} t<\int_{0}^{d_{n_{0}-1}} \phi(t) \mathrm{d} t \leq \int_{0}^{d_{n_{0}}} \phi(t) \mathrm{d} t \leq h_{1} \int_{0}^{d_{n_{0}-1}} \phi(t) \mathrm{d} t<\int_{0}^{d_{n_{0}-1}} \phi(t) \mathrm{d} t,
$$

which is a contradiction. Similarly, if $n_{0}=2 k+1$, then by (2.15) and (2.16)

$$
0<\int_{0}^{d_{n_{0}}} \phi(t) \mathrm{d} t<\int_{0}^{d_{n_{0}-1}} \phi(t) \mathrm{d} t \leq \int_{0}^{d_{n_{0}}} \phi(t) \mathrm{d} t \leq h_{2} \int_{0}^{d_{n_{0}-1}} \phi(t) \mathrm{d} t<\int_{0}^{d_{n_{0}-1}} \phi(t) \mathrm{d} t,
$$

which is also a contradiction. Hence we have

$$
d_{n+1}<d_{n} \text { for all } n \in N \bigcup\{0\} .
$$

Therefore there exists $u \geq 0 \quad \lim _{n \rightarrow \infty} x_{n}=u$. If $u>0$, then

$$
0<\int_{0}^{u} \phi(t) \mathrm{d} t=\lim _{n \rightarrow \infty} \int_{0}^{d_{2 n}} \phi(t) \mathrm{d} t \leq \lim _{n \rightarrow \infty} h_{1} \int_{0}^{d_{2 n-1}} \phi(t) \mathrm{d} t \leq h_{1} \int_{0}^{u} \phi(t) \mathrm{d} t<\int_{0}^{u} \phi(t) \mathrm{d} t,
$$

which is a contradiction. Therefore, $u=0$, i.e., $\lim _{n \rightarrow \infty} d_{n}=0$.

We claim that $\left\{x_{n}\right\}$ is Cauchy. Otherwise, just as the line of proof of Theorem 2.1, there exists $\varepsilon>0$ such that for $k \in N$ there exist $m(k), n(k) \in N$ with $m(k)>n(k)$ such that the parity of $m(k)$ and $n(k)$ is different and (2.11) holds.

If $m(k)$ is even and $n(k)$ is odd, then by Lemma 2.1, (2.11), (2.13) and (iii),

$$
\begin{aligned}
& 0<\int_{0}^{\varepsilon} \phi(t) \mathrm{d} t=\lim _{k \rightarrow \infty} \int_{0}^{d\left(x_{m(k)+1}, x_{n(k)+1}\right)} \phi(t) \mathrm{d} t=\lim _{k \rightarrow \infty} \int_{0}^{d\left(f x_{m(k)}, g x_{n(k)}\right)} \phi(t) \mathrm{d} t \\
& \leq \lim _{k \rightarrow \infty} \psi\left(\int_{0}^{d\left(x_{m(k)}, x_{n(k)}\right)} \phi(t) \mathrm{d} t, \int_{0}^{d\left(x_{m(k)}, f x_{m(k)}\right)} \phi(t) \mathrm{d} t, \int_{0}^{d\left(x_{n(k)}, g x_{n(k)}\right)} \phi(t) \mathrm{d} t, \int_{0}^{d\left(x_{m(k)}, g x_{n}(k)\right)} \phi(t) \mathrm{d} t, \int_{0}^{d\left(f x_{m(k)}, x_{n(k)}\right)} \phi(t) \mathrm{d} t\right) \\
& \leq \psi\left(\int_{0}^{\varepsilon} \phi(t) \mathrm{d} t, 0,0, \int_{0}^{\varepsilon} \phi(t) \mathrm{d} t, \int_{0}^{\varepsilon} \phi(t) \mathrm{d} t\right)<\int_{0}^{\varepsilon} \phi(t) \mathrm{d} t .
\end{aligned}
$$

This is a contradiction. Similarly, we obtain the same contradiction for the case that $m(k)$ is odd and $n(k)$ is even. Therefore, $\left\{x_{n}\right\}$ is a Cauchy sequence. Let $\lim _{n \rightarrow \infty} x_{n}=x^{*}$.

If $f x^{*} \neq x^{*}$, then $d\left(f x^{*}, x^{*}\right)>0$, hence by Lemma 2.1 and (2.13) and (iii),

$$
\begin{aligned}
& 0<\int_{0}^{d\left(f x^{*}, x^{*}\right)} \phi(t) \mathrm{d} t=\lim _{n \rightarrow \infty} \int_{0}^{d\left(f x^{*}, g x_{2 n+1}\right)} \phi(t) \mathrm{d} t \\
& \leq \lim _{n \rightarrow \infty} \psi\left(\int_{0}^{d\left(x^{*}, x_{2 n+1}\right)} \phi(t) \mathrm{d} t, \int_{0}^{d\left(x^{*}, f x^{*}\right)} \phi(t) \mathrm{d} t, \int_{0}^{d\left(x_{2 n+1}, g x_{2 n+1}\right)} \phi(t) \mathrm{d} t, \int_{0}^{d\left(x^{*}, g x_{2 n+1}\right)} \phi(t) \mathrm{d} t, \int_{0}^{d\left(f x^{*}, x_{2 n+1}\right)} \phi(t) \mathrm{d} t\right) \\
& =\psi\left(0, \int_{0}^{d\left(x^{*}, f x^{*}\right)} \phi(t) \mathrm{d} t, 0,0 \int_{0}^{d\left(x^{*}, f x^{*}\right)} \phi(t) \mathrm{d} t\right)<\int_{0}^{d\left(x^{*}, f x^{*}\right)} \phi(t) \mathrm{d} t
\end{aligned}
$$

which is a contradiction, hence $f x^{*}=x^{*}$. Similarly, we obtain $g x^{*}=x^{*}$. Therefore, $x^{*}$ is a common fixed point of $f$ and $g$.

If $y^{*}$ is another common fixed point of $f$ and $g$, then $d\left(x^{*}, y^{*}\right)>0$, hence by (2.13) and (iii),

$$
\begin{aligned}
& 0<\int_{0}^{d\left(x^{*}, y^{*}\right)} \phi(t) \mathrm{d} t=\int_{0}^{d\left(f x^{*}, g y^{*}\right)} \phi(t) \mathrm{d} t \\
& \leq \psi\left(\int_{0}^{d\left(x^{*}, y^{*}\right)} \phi(t) \mathrm{d} t, \int_{0}^{d\left(x^{*}, f x^{*}\right)} \phi(t) \mathrm{d} t, \int_{0}^{d\left(y^{*}, g y^{*}\right)} \phi(t) \mathrm{d} t, \int_{0}^{d\left(x^{*}, g y^{*}\right)} \phi(t) \mathrm{d} t, \int_{0}^{d\left(f x^{*}, y^{*}\right)} \phi(t) \mathrm{d} t\right) \\
& =\psi\left(\int_{0}^{d\left(x^{*}, y^{*}\right)} \phi(t) \mathrm{d} t, 0,0, \int_{0}^{d\left(x^{*}, y^{*}\right)} \phi(t) \mathrm{d} t, \int_{0}^{d\left(x^{*}, y^{*}\right)} \phi(t) \mathrm{d} t\right)<\int_{0}^{d\left(x^{*}, y^{*}\right)} \phi(t) \mathrm{d} t .
\end{aligned}
$$

This is a contradiction. Hence $x^{*}$ is the unique common fixed point of $f$ and $g$.

Using Theorem 2.3 and the Example 2.2, we have the next result.

Theorem 2.4. Let $(X, d)$ be a complete metric space, $f, g: X \rightarrow X$ two mappings. If

$$
\ln ^{(1+d(f x, g y))} \leq \psi\left(\ln ^{(1+d(x, y))}, \ln ^{(1+d(x, f x))}, \ln ^{(1+d(y, g y))} \ln ^{(1+d(x, g y))}, \ln ^{(1+d(f x, g))}\right) \text { for all } x, y \in X
$$

where $\psi \in \Psi$. Then $f$ and $g$ have a unique common fixed point $u$. 
Combining Theorem 2.4 and Example 2.1, we obtain the following result.

Theorem 2.5. Let $(X, d)$ be a complete metric space, $f, g: X \rightarrow X$ two mappings. If for each $x, y \in X$,

$$
1+d(f x, g y) \leq(1+d(x, y))^{a_{1}}(1+d(x, f x))^{a_{2}}(1+d(y, g y))^{a_{3}}(1+d(x, g y))^{a_{4}}(1+d(f x, y))^{a_{5}}
$$

where $a_{i} \geq 0$ for all $i=1, \cdots, 5$ and $a_{1}+a_{2}+a_{3}+2 a_{4}+2 a_{5}<1$. Then $f$ and $g$ have a unique common.

\section{Acknowledgements}

The research is partially supported by the National Natural Science of Foundation of China (No. 11361064).

\section{References}

[1] Banach, A. (1929) Sur les opérations dans les ensembles abstraits et leur application aux équations intégrales. Fundamenta Mathematicae, 3, 133-181.

[2] Aliouche, A. (2006) A Common Fixed Point Theorem for Weakly Compatible Mappings in Symmetric Spaces Satisfying a Contractive Condition of Integral Type. Journal of Mathematical Analysis and Applications, 322, 796-802. http://dx.doi.org/10.1016/j.jmaa.2005.09.068

[3] Altun, I. and Türkoğlu, D. (2009) Some Fixed Point Theorems for Weakly Compatible Mapping Satisfying an Implicit Relation. Taiwanese Journal of Mathematics, 13, 1291-1304.

[4] Jachymski, J. (2009) Remarks on Contractive Conditions of Integral Type. Nonlinear Analysis, 71, 1073-1081. http://dx.doi.org/10.1016/j.na.2008.11.046

[5] Mocanu, M. and Popa, V. (2008) Some Fixed Point Theorems for Mappings Satisfying Implicit Relations in Symmetric Spaces. Libertas Mathematica, 28, 1-13.

[6] Gairola, U.C. and Rawat, A.S. (2008) A Fixed Point Theorem for Integral Type Inequality. International Journal of Mathematical Analysis, 2, 709-712.

[7] Moradi, S. and Omid, M. (2010) A Fixed Point Theorem for Integral Type Inequality Depending on Another Function. International Journal of Mathematical Analysis, 4, 1491-1499.

[8] Altun, I., Abbas, M. and Simsek, H. (2011) A Fixed Point Theorem on Cone Metric Spaces with New Type Contractivity. Banach Journal of Mathematical Analysis, 5, 15-24. http://dx.doi.org/10.15352/bjma/1313362998

[9] Popa, V. and Mocanu, M. (2009) Altering Distance and Common Fixed Points under Implicit Relations. Hacettepe Journal of Mathematics and Statistics, 38, 329-337.

[10] Abbas, M. and Rhoades, B.E. (2007) Common Fixed Point Theorems for Hybrid Pairs of Occasionally Weakly Compatible Mappings Satisfying Generalized Contractive Condition of Integral Type. Fixed Point Theory and Applications, 2007, Article ID: 054101. http://dx.doi.org/10.1155/2007/54101

[11] Rakotch, E. (1962) A Note on Contractive Mappings. Proceedings of the American Mathematical Society, 13, $459-465$. http://dx.doi.org/10.1090/S0002-9939-1962-0148046-1

[12] Branciari, A. (2002) A Fixed Point Theorem for Mappings Satisfying a General Contractive Condition of Integral Type. International Journal of Mathematics and Mathematical Sciences, 29, 531-536. http://dx.doi.org/10.1155/S0161171202007524

[13] Liu, Z.Q., Li, X., Kang, S.M. and Cho, S.Y. (2011) Fixed Point Theorems for Mappings Satisfying Contractive Conditions of Integral Type and Applications. Fixed Point Theory and Applications, 2011, 64. http://dx.doi.org/10.1186/1687-1812-2011-64

[14] Abbas, M., Cho, Y.J. and Nazir, T. (2012) Common Fixed Points of Ćirić-Type Contractive Mappings in Two Ordered Generalized Metric Spaces. Fixed Point Theory and Applications, 2012, 139. http://dx.doi.org/10.1186/1687-1812-2012-139

[15] Gu, F. and Ye, H.Q. (2012) Common Fixed Point Theorems of Altman Integral Type Mappings in Metric Spaces. Abstract and Applied Analysis, 2012, Article ID: 630457. http://dx.doi.org/10.1155/2012/630457 